index ecomunicación | no 10(1) 2020 | Páginas 149-172

E-ISSN: 2174-1859 | ISSN: 2444-3239 | Depósito Legal: M-19965-2015

Recibido el 04_03_2020 | Aceptado el 25_04_2020 | Publicado el 15_10_2020

\title{
GÉNERO Y PERFIL EN LAS AUTORÍAS Y COLABORACIONES DE 'REVISTA MEDITERRÁNEA DE COMUNICACIÓN' (2010-2019)
}

GENDER AND PROFILE IN THE AUTHORSHIPS AND COLLABORATIONS OF 'MEDITERRANEAN JOURNAL OF COMMUNICATION' (2010-2019)

https://doi.org/10.33732/ixc/10/01Genero

Jesús Segarra-Saavedra
$\begin{array}{r}\text { Universidad de Alicante } \\ \text { jesus.segarra@ua.es }\end{array}$
orcid.org/0000-0001-9420-5258

Victoria Tur-Viñes

Universidad de Alicante victoria.tur@ua.es

orcid.org/0000-0002-8442-8068

\section{Tatiana Hidalgo-Marí \\ Universidad de Alicante \\ tatiana.hidalgo@ua.es \\ orcid.org/0000-0003-4599-5876}


150

Resumen: Se presenta un estudio descriptivo y retrospectivo, bajo la perspectiva de género, centrado principalmente en el perfil de autoría, pero también en el de colaboradores/as de Revista Mediterránea de Comunicación $(R M C)$ durante sus 10 años de existencia. El objetivo es visibilizar la relevancia, rol y contribución de la mujer al conocimiento en el ámbito de la comunicación. Para ello, se utiliza una muestra formada por 414 autorías de 238 artículos y 30 coordinaciones de 17 monográficos sobre la que se aplican las siguientes variables: perfil sociodemográfico (nombre, apellidos y género), institucional (institución y procedencia geográfica), investigador (título, idioma/s, resumen y grado de doctor/a), perfil en Google Scholar (citas, índice H e índice H-10) y presencia en Orcid. Los resultados indican que la mujer adquiere protagonismo, tanto como autora de artículos (rol de producción) como coordinadora de monográficos (rol de organización), pero los hombres son más citados que las mujeres (rol de liderazgo).

Palabras clave: autoría; comunicación científica; género; Google Scholar; revistas científicas; Revista Mediterránea de Comunicación.

Abstract: A descriptive and retrospective study is presented, from a gender perspective, focusing mainly on the profile of authorship but also on that of collaborators of the Mediterranean Journal of Communication (RMC) during its 10 years of existence. The objective is to make visible the relevance, role and contribution of women to knowledge in the field of Communication. The sample: 414 authors of 238 articles and 30 coordinators of 17 monographs are analyzed. Dimensions and variables: sociodemographic profile (name, surname and gender), institutional profile (institution and geographical origin), researcher (title, language/s, abstract and $\mathrm{PhD}$ degree), Google Scholar profile (citations, $\mathrm{H}$ index and $\mathrm{H}-10$ index) and presence in Orcid. Results: the woman acquires protagonism, both as author of articles (production role) and coordinator of monographs (organization role) but men are more cited than women (leadership role).

Keywords: Autorship; Scientific Communication; Scientific; Gender; Google Scholar; Scientific Journals; Mediterranean Journal of Communication. 


\section{Introducción}

El protagonismo de la mujer en el mundo académico se ha producido de forma lenta y paulatina a lo largo del tiempo. En comparación con el protagonismo ostentado por los hombres, las mujeres se incorporan primero a las aulas, después al profesorado y, todavía hoy, siguen conquistando la esfera de la alta gestión académica. Los hombres han tenido una presencia natural y normalizada, desde los orígenes, en todas las esferas. El rol social tradicional de la era moderna ha situado a las mujeres, prioritariamente, en la esfera doméstica familiar y, al hombre, en el exterior del hogar, impulsando el resto de las actividades, también en el ámbito educativo.

Si bien es cierto que la primera mitad del siglo XX se caracterizó por el acceso de la mujer a la universidad, esta incorporación se puede entender como un intento fallido en la consolidación de la presencia femenina en la institución académica. Aunque progresivamente, a medida que avanzaba el siglo XX, las mujeres iban adquiriendo mayor visibilidad en la universidad, se debe esperar a las últimas décadas del siglo XX para poder hablar de una incorporación paulatina pero constante a la esfera pública en general y al contexto académico superior en particular. En este sentido, los últimos años del siglo XX se caracterizan por el incremento paulatino que experimenta la incorporación de la mujer a la universidad y su participación en la economía del conocimiento, una esfera de la que había estado totalmente apartada prácticamente desde los orígenes de las universidades en España.

Que la incorporación de la mujer a la esfera académica y científica ha sido tardía y lenta es una evidencia que han demostrado estudios tanto históricos como aplicados. Sin embargo, mirando con perspectiva, se puede decir que se ha desarrollado un camino sólido y consolidado desde dicha incorporación, en ambas dimensiones - cuantitativa y cualitativa-, gracias al reconocimiento progresivo que se ha llevado tanto desde las instituciones como desde la propia demanda social (Vega y Santos, 2010).

A pesar del interés social y de la importancia de los estudios de género en los últimos tiempos, lo cierto es que el estudio de la mujer científica no ha suscitado demasiado interés en España, ni en el resto de los países iberoamericanos. Algunas investigaciones en el ámbito español son las llevadas a cabo por Alcalá (1996), Magallón (1999) o Santesmases (2000), en un recorrido que nos lleva desde las pioneras de principios de siglo a la presencia en nuestros días de españolas en la investigación u otros campos profesionales, de corte más social, que sitúan en el problema de conciliación la desigualdad en 
cifras entre hombres y mujeres alrededor de la ciencia (Almodóvar, 1996; Agudo, 2006).

El estudio sobre la incorporación de las mujeres a la esfera universitaria ha sido un tema de estudio recurrente, en especial en las últimas décadas del siglo XX. Estas investigaciones han priorizado el enfoque de género sobre la docencia y la gestión en la institución académica desde distintas perspectivas, pero poco se ha profundizado en la actividad de las mujeres y sus contribuciones a la actividad investigadora y sus aportaciones científicas. Esta área de estudio se encuentra en fase inicial y cobra especial auge en los últimos años con el impulso de estudios enfocados a conocer la participación y el desarrollo femenino en el conocimiento científico.

El presente trabajo tiene como finalidad explorar la evolución de la participación de hombres y mujeres con motivo del x aniversario de Revista Mediterránea de Comunicación/Mediterranean Journal of Communication. Mediante el estudio de caso de esta revista, se pretende visibilizar las diferencias entre hombres y mujeres a la hora de publicar y difundir el conocimiento, así como colaborar en las tareas de edición científica. Esta aproximación abunda en el estudio de la economía del conocimiento en Ciencias de la Comunicación desde la perspectiva de la producción científica y la participación en la creación del conocimiento académico y científico.

\section{Marco teórico}

Cuando en los años ochenta se popularizó el término 'techo de cristal' o glass ceiling (Fong, Hung \& Li, 2005) para denominar a las barreras sutiles casi invisibles que limitan y dificultan el acceso de la mujer a posiciones de poder 0 prestigio en cualquier contexto laboral, se dio visibilidad a un fenómeno que, desde hacía siglos, venía perpetuando la hegemonía del hombre sobre la mujer en la gran mayoría de esferas laborales (Guil Bozal, 2007) y, en concreto, en el entorno científico y universitario, que es el que se trata en este trabajo.

Investigaciones como las de Peñasco (2005) y Valls (2008) confirman que el acoso laboral y la violencia de género, así como las luchas de poder y la competitividad, continúan siendo las principales fuentes de deterioro del ambiente universitario (Aguilar, Alonso, Melgar y Molina, 2009) a pesar del progreso hacia la igualdad. Resulta necesario destacar las dificultades adicionales ('techo de cristal') que se encuentran las mujeres a la hora de promocionar a puestos de liderazgo ocupados por hombres, de forma reiterada. También merecen mención los profundos impedimentos a los que se enfrentan para acceder a fondos que financien sus proyectos de investigación (García de Cortázar y García de León, 2001). Según Vega y Santos (2010: 131): 
Cierto es que se produjeron algunos progresos cuando a partir de los años ochenta empezó a crecer el número de profesoras, investigadoras y catedráticas, pero parece que no se avanza decididamente hacia una situación igualitaria en la distribución de los principales cargos, sobre todo en lo que se refiere a la obtención de cátedras, cuyas cifras crecen tan lentamente que a veces parecen estancarse.

Resulta necesario, llegado este punto, plantearse cómo es el acceso de la mujer a la ciencia y a la producción científica; si se tiene en cuenta que la investigación, la publicación de resultados y la transferencia del conocimiento son los pilares esenciales para poder acreditar y, posteriormente, acceder a posiciones líderes en el contexto de la investigación científica. En este sentido, merece la pena destacar el texto de Clair (1996) citado por González y Pérez (2002), que recoge las investigaciones de la IV Conferencia Mundial de las Naciones Unidas sobre la Mujer (Pekín 1995). En el mismo documento, los resultados expuestos en la obra editada de Clair (1996) sobre la conferencia: «[...] apuntaban en general al estancamiento (en algunos casos, incluso regresión) de la participación de las mujeres en las actividades científicas tecnológicas en comparación con 1985, año de la III Conferencia de Nairobi» (2002: 5).

Como se ha venido esbozando, la incorporación de la mujer al contexto científico y académico ha sido más lento, escalonado y complejo (Alic, 1981) debido a los múltiples factores socioculturales que han marcado su rol alrededor de la investigación y la producción científica. Estos obstáculos han afectado, como es obvio, a la publicación académica, que es uno de los requisitos imprescindibles, no solo para la visibilidad de los resultados de investigación, sino también para la promoción en la carrera universitaria.

En este sentido, la publicación académica se relaciona directamente con el mecanismo de promoción, contratación, acceso y reconocimiento profesional establecido en cada sistema universitario, con las variaciones particulares que cada autonomía y universidad le hayan querido imprimir. A cada texto científico se le atribuye la capacidad de contribuir (Blackburn \& Lawrence 1995), en menor o mayor medida, al conocimiento de un campo. De hecho, las posibles publicaciones son sometidas a procesos de revisión y selección con este fin: identificar si existe aportación (novedad, originalidad y/o avance) y esta es cualitativamente aceptable (demuestra conocimiento de las aportaciones previas y las cita, aplica la metodología con rigor, selección con criterios de representatividad de las unidades de estudio - sujeto, contenidos, empresas y datos, entre otros-, análisis correcto y exhaustivo de los resultados y conclusiones reveladoras).

Esa estrecha vinculación entre publicación y carrera académica se ha reforzado en España con el programa de acreditación del profesorado de la 
Agencia Nacional de Evaluación de la Calidad y Acreditación (ANECA). El artículo científico se erige como mérito preferente de valoración, determinante para conseguir la promoción académica. La publicación académica, más que cualquier otra cosa, es la medida de la productividad de la investigación del profesorado universitario. En las humanidades, la monografía es la publicación más frecuente, pero, en las ciencias puras y en muchas ciencias sociales, son los artículos de revistas científicas. Actualmente, el alumnado de doctorado tiene que sumar a su tesis doctoral publicaciones para poder ser contratado por una universidad. Y una vez que son contratados, generalmente se requieren más publicaciones en revistas líderes en el área para llegar a ser titulares.

El progreso de las mujeres en el mundo académico ha sido durante mucho tiempo un tema candente, sobre todo el debate sobre por qué las mujeres publican menos que los hombres. Las profesoras tienen más probabilidades de enfatizar la calidad sobre la cantidad produciendo menos textos, pero de mayor calado, que sus colegas masculinos. Además, los estudios muestran que las mujeres dedican menos tiempo a la investigación y más tiempo a la docencia o a la gestión (Wilson, 2012). A menudo, son las investigaciones y publicaciones, que requieren una atención sostenida e intensa las que sufren cuando las mujeres dedican tiempo al cuidado de los niños pequeños.

Sin embargo, a medida que más mujeres obtienen doctorados y acceden a puestos docentes, se reduce la brecha entre el número de mujeres y hombres en la academia. Sirvan como ejemplo la comparativa temporal de datos: mientras que el Instituto Nacional de Estadística, INE (2019) indica que el $41,3 \%$ del profesorado universitario son mujeres, el Instituto de la Mujer (2009) recogía que las mujeres representaban en el curso 2006/2007 el $36,05 \%$. Sigue abierto el debate acerca de si se puede o se debe hacer algo sobre las diferencias de género que permanecen en la publicación, contratación, promoción y remuneración. La diatriba existente en la investigación de género plantea discernir si esas diferencias son resultado de las elecciones naturales y espontáneas que hacen las mujeres o son consecuencia de algún tipo de discriminación o dificultad.

Si nos aproximamos a datos concretos sobre la productividad de las mujeres, el promedio de publicaciones de las académicas es inferior al de los hombres, como se ha evidenciado en la literatura previa sobre el tema en la década de los 90 (Blackburn \& Lawrence, 1995; Creamer, 1998; Dinauer \& Ondeck, 1999; Buffalino-Roland \& Fontanesi-Seime, 1996; Schneider, 1998). Entre los factores que afectan a la publicación regular, se encuentran la ambición, la reputación, el mérito, el apoyo institucional y los recursos, las redes 
profesionales, las relaciones de mentorización, las redes de trabajo con otros colegas, el tema, la metodología de investigación y el tiempo, según los estudios de Mathews \& Andersen (2001).

Aunque el porcentaje de autoras todavía es menor que la representación general de las mujeres en el personal docente e investigador (PDI) a tiempo completo de las facultades, Wilson (2012) detecta que esa brecha disminuye a medida que más mujeres ingresan al profesorado. También determinó que las mujeres se agrupan en ciertos subcampos científicos y están poco representadas en la posición de primer firmante, acentuándose esta diferencia en las áreas biológicas, por ejemplo.

En otras colaboraciones relacionadas con el proceso editorial, las mujeres también tienen una representación no equitativa. Utilizando la información de acceso público sobre la identidad de 9.000 editores y 43.000 revisores de Frontiers (editorial suiza de publicaciones de acceso abierto), Helmer, Schottdorf, Neef \& Battaglia (2017) demostraron que las mujeres están subrepresentadas en el proceso de revisión por pares, que los editores de ambos sexos operan con preferencia sustancial del mismo género (homofilia), y que los mecanismos de esta homofilia dependen del género. También revelaron que la homofilia persiste incluso si se alcanza la paridad numérica entre géneros, destacando la necesidad de mayores esfuerzos para combatir formas más sutiles de sesgo de género en la publicación académica. La homofilia está generalizada entre los editores hombres mientras que entre las editoras mujeres existen pocas con tendencia a la homofilia pero con niveles mucho más altos que los hombres, es decir, las editoras homofílicas son menos en número pero la practican de forma más intensa.

El estudio contempló el perfil de 9.000 editores, 43.000 revisores y 126.000 autores en 14.000 artículos publicados en 142 revistas de Ciencias, Salud, Ingeniería, Humanidades y Ciencias Sociales reflejando los siguientes porcentajes de mujeres: $39 \%$ autoras, $30 \%$ revisoras y $28 \%$ editoras. Helmer et al. (2017) sugieren la automatización de los procesos de selección de revisores, el anonimato del nombre de cualquier participante en el proceso editorial y la presencia continua de estadísticas de estos procesos para que la información disponible sobre género permita tomar conciencia y corregir desigualdades que, de otro modo, involuntariamente se perpetúan.

Los estudios previos sobre el equilibrio de género en el proceso de revisión por pares son escasos, basados en pequeños conjuntos de datos con carácter monodisciplinario y parcialmente contradictorios en sus conclusiones (Lloyd, 1990; Gilbert, Williams \& Lundberg, 1994; Budden, Tregenza, Aarssen, Koricheva, Leimu \& Lortie, 2008; Borsuk, Aarssen, Budden, Koricheva, Leimu, 
Tregenza, \& Lortie, 2009; Knobloch-Westerwick, Carrol \& Huge, 2013; Larivière, Ni, Gingras, Cronin, \& Sugimoto, 2013; Buckley, Sciligo, Adair, Case, \& Monks, 2014; Demarest, Freeman, \& Sugimoto, 2014; Handley, Frantz, Kocovsky, De Vries, Cooke, \& Claussen, 2015; Fox, Burns, \& Meyer, 2016). Las características del proceso de revisión más utilizado (doble par anónimo) exigen que las identidades tanto de los revisores como de los autores sean confidenciales, aspecto que influye directamente en la investigación de género de los revisores.

A la vista de los datos expuestos, se puede afirmar que la producción científica académica presenta diferencias de género importantes. Las iniciativas para corregir esta inequidad responden a dos perspectivas. En primer lugar, a la transversalización de género: se orienta a largo plazo y promueve la consideración igualitaria de ambos géneros en todos los niveles, de abajo a arriba, en cada proceso de toma de decisiones. Se basa en la motivación y la concienciación (Woodward, 2008). En segundo lugar, la cuota, un instrumento tecnocrático para luchar contra las diferencias de género en clara estrategia de arriba-abajo, recibe críticas porque puede proporcionar una coartada para no modificar las actitudes en profundidad (Squires, 2008).

En el contexto de las Ciencias Sociales y, particularmente, en el seno de los estudios de Comunicación, conocer la aportación femenina al avance del conocimiento científico no solo se vuelve indispensable desde un punto de vista antropológico, sino que también es clave para detectar aspectos vinculados al liderazgo investigador, la capacidad productiva y la propia construcción del discurso científico que se desarrolla por parte de las mujeres. Así pues, a pesar de las numerosas dificultades y contrariedades, cada vez más la mujer va adquiriendo el protagonismo por el que ha luchado durante tantos años, no solo en el ámbito público sino también en el social.

\section{Objetivos y metodología}

Este estudio descriptivo longitudinal utiliza el estudio de caso para tratar de determinar la participación de las mujeres como creadoras, investigadoras, difusoras y evaluadoras del conocimiento científico.

El estudio de caso se focaliza en Revista Mediterránea de Comunicación / Mediterranean Journal of Communication (en adelante, RMC), una publicación científica avalada, entre otros, por el sello de calidad de la Fundación Española para la Ciencia y la Tecnología (FECYT, 2016-2019), por estar incluida en Emerging Source Citation Index (ESCI) de Clarivate desde 2015, donde el ranking ESCI de Revista Comunicar (2019) le otorga la 2 ${ }^{\text {a }}$ posición en España y la $20^{\mathrm{a}}$ en el mundo en la categoría Communication (Q1, IF: 0,730). Es revista 
fuente en Dialnet Métricas (2018), 5a en España (Q1, 2018), 5a en Iberoamérica en el ranking REDIB (CSIC) y 8 ${ }^{-}$de 41 en Google Scholar Metric (Google Académico) en el campo de Comunicación. Motiva este estudio el hecho de que en el último número publicado en julio de 2019 (10.2) se constatara una presencia ligeramente superior de 19 mujeres (51\%) frente a los 18 hombres (49\%) en autorías.

El artículo tiene como objetivo general aportar datos concretos sobre el papel de la mujer como investigadora y científica dentro de $R M C$, en concreto, analizando su perfil y roles dentro de la revista. De este objetivo general surgen tres objetivos específicos, concretos, que se corresponden con la operatividad del trabajo. El primer objetivo específico (OE1) pretende analizar el perfil sociodemográfico, institucional, académico e investigador de autoría en $R M C$. Para ello, se plantea un análisis de contenido (ver tabla 1) comparativo de ambos géneros a partir de la información recibida en el propio sistema de la revista (OJS) en el período comprendido entre 2010 y 2019.

Dicho análisis recoge las siguientes variables: perfil sociodemográfico e investigador, institución de afiliación, carácter nacional o internacional, grado académico, roles más utilizados (coordinación o autoría), tipo de textos más publicados (editoriales, artículos, ensayos o reseñas), temáticas e idiomas que firman con más frecuencia, índice de coautoría, e implicación de la autoría en el proceso editorial que trata de internacionalizar la producción científica a través de la traducción de los textos a otros idiomas, especialmente el inglés. Todas estas variables permiten ofrecer una taxonomía o perfil de investigadores e investigadoras en Ciencias de la Comunicación, que pretende ser extrapolable a otras revistas de la misma área, con el fin de asentar las bases de un estudio más amplio que aborde la contribución de las mujeres al conocimiento científico en Comunicación, así como su participación estimulante en la actividad científica-investigadora y su labor en la difusión el conocimiento.

Tabla 1: Variables referentes al 01

\begin{tabular}{|c|l|c|l|}
\hline \multicolumn{4}{|c|}{ Objetivo específico 1 (OE1). Variables analizadas } \\
\hline V1 & ID & V11 & Nombre y apellidos \\
\hline V2 & ID de OJS (Open Journal System) & V12 & Género \\
\hline V3 & Año & V13 & Posesión del grado de doctor/a \\
\hline V4 & Volumen & V14 & No total de autores/as \\
\hline V5 & Número & V15 & Orden de autoría \\
\hline V6 & Título & V16 & Institución de afiliación \\
\hline V7 & Palabras clave & V17 & $\begin{array}{l}\text { Procedencia nacional/ } \\
\text { internacional }\end{array}$ \\
\hline
\end{tabular}




\begin{tabular}{|r|l|r|l|}
\hline V8 & $\begin{array}{l}\text { Tipo de texto: editorial, artículo, } \\
\text { ensayo o reseña }\end{array}$ & V18 & País \\
\hline V9 & Idioma/s & & \\
\cline { 1 - 2 } V10 & $\begin{array}{l}\text { Aportación de traducción del texto } \\
\text { original }\end{array}$ & & \\
\hline
\end{tabular}

Fuente: elaboración propia

El segundo objetivo específico (OE2) pretende analizar tanto la presencia en las redes académicas Google Académico y Orcid como el alcance en Google Académico, de cada autoría en $R M C$. Para ello, se utilizan las variables quedan recogidas en la tabla 2 . Con este segundo objetivo se pretende evaluar las diferencias de género en la gestión de la visibilidad de las producciones personales mediante la existencia de perfiles básicos como GS y Orcid.

Tabla 2: Variables referentes al 02

\begin{tabular}{|c|l|c|l|}
\hline \multicolumn{3}{|c|}{ Objetivo específico 2 (OE2): variables analizadas } \\
\hline V19 & Presencia en GS & V23 & Presencia en Orcid \\
\hline V20 & Número total de citas & V24 & Perfil completado \\
\hline V21 & Índice H & \multicolumn{3}{|c}{} \\
\hline V22 & Índice H-10 & \multicolumn{3}{|c|}{} \\
\hline
\end{tabular}

Fuente: elaboración propia

Además, el estudio aplica la perspectiva de género al análisis de los miembros del comité editorial y a participantes tanto en el comité internacional de edición como en el comité científico internacional y de revisores/as, que será objeto de estudio en el OE3. A partir de estos objetivos, se toman diversas unidades de análisis: sobresalen el artículo y la autoría, pero también se utiliza cada volumen y número, así como cada miembro del equipo de trabajo de $R M C$.

\section{Resultados}

En relación al OE1, durante los diez primeros años de vida de $R M C$ se contabilizan un total de 303 textos, de los cuales priman los artículos publicados $(78,5 \%)$ bien en la sección «monográfico» o en «miscelánea», seguidos de las reseñas de libros y artículos $(9,6 \%)$, los editoriales por número (firmados por la editora o la coeditora de $R M C$ ) y por monográfico (firmados por los/as coordinadores/as de este) y los ensayos de firmas invitadas (2,6\%). 
Tabla 3: Publicaciones por año, volumen, número y tipo

\begin{tabular}{|c|c|c|c|c|c|c|c|c|c|c|}
\hline \multirow[b]{2}{*}{ Año } & \multirow[b]{2}{*}{ Vol. } & \multicolumn{2}{|c|}{$\mathrm{N}^{\circ}$} & \multicolumn{3}{|c|}{ Monográficos } & \multicolumn{4}{|c|}{ Publicaciones } \\
\hline & & 1 & 2 & No & $\begin{array}{l}\text { Coord. } \\
\text { (M) }\end{array}$ & $\begin{array}{l}\text { Coord. } \\
\text { (H) }\end{array}$ & Editoriales & Artículos & Ensayos & Reseñas \\
\hline \multirow{2}{*}{2019} & 10 & 1 & & 2 & 3 & 1 & 3 & 16 & 0 & 3 \\
\hline & & & 1 & 2 & 4 & 1 & 3 & 17 & 0 & 5 \\
\hline \multirow{2}{*}{2018} & 9 & 1 & & 2 & 1 & 2 & 3 & 30 & 0 & 4 \\
\hline & & & 1 & 2 & 1 & 3 & 3 & 21 & 0 & 5 \\
\hline \multirow{2}{*}{2017} & 8 & 1 & & 1 & 0 & 1 & 2 & 13 & 0 & 2 \\
\hline & & & 1 & 2 & 3 & 1 & 3 & 18 & 0 & 1 \\
\hline \multirow{2}{*}{2016} & 7 & 1 & & 1 & 0 & 1 & 2 & 13 & 0 & 0 \\
\hline & & & 1 & 1 & 1 & 1 & 2 & 18 & 0 & 0 \\
\hline \multirow{2}{*}{2015} & 6 & 1 & & 0 & - & - & 1 & 6 & 4 & 1 \\
\hline & & & 1 & 1 & 0 & 1 & 2 & 12 & 0 & $\underline{0}$ \\
\hline \multirow{2}{*}{2014} & 5 & 1 & & 1 & 1 & 1 & 2 & 10 & 2 & 2 \\
\hline & & & 1 & 1 & 1 & 0 & 2 & 10 & 0 & 2 \\
\hline \multirow{2}{*}{2013} & 4 & 1 & & 0 & - & - & 0 & 10 & 1 & 2 \\
\hline & & & 1 & 1 & 1 & 1 & 0 & 10 & 0 & 2 \\
\hline \multirow{2}{*}{2012} & 3 & 1 & & 0 & - & - & 0 & 6 & 0 & $\underline{0}$ \\
\hline & & & 1 & 0 & - & - & 0 & 6 & 0 & 0 \\
\hline 2011 & 2 & 1 & & 0 & - & - & 0 & 11 & 1 & 0 \\
\hline 2010 & 1 & 1 & & 0 & - & - & 0 & 11 & 0 & 0 \\
\hline Subtotal & 10 & $\begin{array}{l}1 \\
0\end{array}$ & 7 & 17 & 16 & 14 & 28 & 238 & 8 & 29 \\
\hline \multicolumn{5}{|c|}{ Subtotal (\%) } & 53,3 & 46,7 & 9,2 & 78,5 & 2,6 & 9,6 \\
\hline Total & & & & & & & & & & 303 \\
\hline
\end{tabular}

Fuente: elaboración propia

Se constata, además, la consolidación de la publicación de monográficos de temáticas especializadas, no solo atendiendo al mantenimiento de dicha sección, sino también al incremento en la cantidad de monográficos por número de la revista, que en los últimos cuatro números de $R M C$ se mantiene en dos por número. Lo contrario ocurre con la sección «ensayos», que desde el número 2015.6.1 se extinguió por falta de recepción de este tipo de textos. Además, en lo relativo a los monográficos, existe un mayor porcentaje $(53,3 \%)$ de coordinadoras que de coordinadores $(46,7 \%)$.

Dado que la comunidad académica y las agencias de evaluación nacional y autonómicas priman los artículos científicos, y a la luz de los datos anteriores, el presente estudio se centra en los artículos publicados en $R M C$.

Los/as autores/es con mayor número de artículos son: (cuatro) Carmen Marta-Lazo y Paulo Ribeiro-Cardoso; (tres) Araceli Castelló-Martínez, Enrique Carrasco-Molina e Ignacio Aguaded y (dos) Alieen Stewart, Alfonso Méndiz- 
Noguero, Alicia de-Lara-González, Ana Sedeño-Valdellós, Ángel TorresToukoumidis, Begoña Ivars-Nicolás, Belén Puebla-Martínez, Carmen CristófolRodríguez, Daniel Barredo-Ibáñez, Eliseo Rodríguez-Monteagudo, Eva Campos-Domínguez, Gloria Jiménez-Marín, Javier Gil-Quintana, José-Luis González-Esteban, José-Antonio Gabelas-Barroso, Juan-Ángel Jódar-Marín, Leticia Porto-Pedrosa, Lourdes López-Pérez, Lucía Camarero-Cano, Luis MañasViniegra, Manuel Montes-Vozmediano, Martín Oller-Alonso, Rafael MarfilCarmona, Raymond Colle, Roberto Gelado-Marcos, Sergio Roses y Tatiana Hidalgo-Marí. Representando a cada uno/a de estos-as autores/as como firmantes de un solo texto, se identifican 414 autores/as distintos/as en $R M C$, de los cuales $251(60,6 \%)$ son mujeres y $163(39,4 \%)$ hombres.

En lo referente a la posesión del título de doctor/a, nueve autorías han publicado en $R M C$ antes y después de presentar sus tesis doctorales, evidenciando un interés permanente a lo largo de la carrera universitaria (Alicia deLara-González, Belén Puebla-Martínez, Daniel Barredo-Ibáñez, Eliseo Rodríguez-Monteagudo, Leticia Porto-Pedrosa, Lucía Camarero-Cano, Paulo Ribeiro-Cardos, Rafael Marfil-Carmona y Roberto Gelado-Marcos). En general, 304 autorías $(71,9 \%)$ poseían el título de doctor/a cuando publicaron sus textos en $R M C$ y $119(28,1 \%)$ no. A su vez, de las 304 autorías de doctores, 182 son de mujeres $(59,9 \%)$ y $122(40,1 \%)$ de hombres.

Con respecto al porcentaje de autoría por artículo, el promedio es de 1,9 , siendo cuatro el máximo número alcanzado. Se detecta autoría única en 106 textos, 56 son hombres $(52,8 \%)$ y 50 mujeres $(47,2 \%)$. Como primer firmante se identifican 140 artículos: 82 de mujeres $(65,9 \%)$ y 42 de hombres (34,1\%); 51 texto tienen coautoría de tres $(60,8 \%$ mujeres y $39,2 \%$ hombres) y siete tiene coautoría de cuatro $(71,4 \%$ hombres y $28,6 \%$ mujeres).

En cuanto a la procedencia de las autorías, destaca la nacional $(76,2 \%)$ sobre la internacional $(23,8)$, siendo Argentina y Ecuador (17\% cada uno), Portugal (14\%) y México (10\%), los países con mayor número de aportaciones en forma de artículo.

Atendiendo a las instituciones internacionales desde las que aplican los/as autores/as, destaca la Universidade Fernando Pessoa (7\%) y la Universidad de Buenos Aires y la Universidad de Las Américas (ambas con un 5\%).

Tabla 4: Países e instituciones internacionales que aportan textos a $R M C$

\begin{tabular}{|c|c|c|c|c|}
\hline País & Frecuencia & $\%$ & Universidad & $\%$ \\
\hline \multirow{4}{*}{ Argentina } & \multirow{4}{*}{18} & \multirow{4}{*}{17} & Universidad de Buenos Aires & 5 \\
\hline & & & Universidad Nacional de Avellaneda & 3 \\
\hline & & & Universidad Nacional de Lomas de Zamora & 3 \\
\hline & & & Universidad Nacional de Rosario & 3 \\
\hline
\end{tabular}




\begin{tabular}{|c|c|c|c|c|}
\hline & & & Universidad Nacional de Córdoba & 2 \\
\hline & & & Universidad Austral & 2 \\
\hline \multirow{9}{*}{ Ecuador } & \multirow{9}{*}{18} & \multirow{9}{*}{17} & Universidad de las Américas & 5 \\
\hline & & & Pontificia Universidad Católica del Ecuador & 3 \\
\hline & & & Escuela Superior Politécnica de Chimborazo & 2 \\
\hline & & & Universidad Estatal de Milagro & 2 \\
\hline & & & Instituto de Altos Estudios Nacionales & 1 \\
\hline & & & Universidad Politécnica Salesiana & 1 \\
\hline & & & Universidad San Francisco de Quito & 1 \\
\hline & & & Universidad Técnica de Cotopaxi & 1 \\
\hline & & & Universidad Técnica Particular de Loja & 1 \\
\hline \multirow{9}{*}{ Portugal } & \multirow{9}{*}{15} & \multirow{9}{*}{14} & Universidade Fernando Pessoa & 7 \\
\hline & & & Instituto Politécnico de Coimbra & 1 \\
\hline & & & Instituto Politécnico de Viana do Castelo & 1 \\
\hline & & & Instituto Portugués de Administración de Marketing & 1 \\
\hline & & & $\begin{array}{l}\text { Instituto Superior de Contabilidad y Administración } \\
\text { de Oporto }\end{array}$ & 1 \\
\hline & & & Universidad de Oporto & 1 \\
\hline & & & Universidade da Beira Interior & 1 \\
\hline & & & Universidade do Minho & 1 \\
\hline & & & Universidade Nova de Lisboa & 1 \\
\hline \multirow{8}{*}{ México } & \multirow{8}{*}{11} & \multirow{8}{*}{10} & Universidad Anáhuac & 2 \\
\hline & & & Universidad de Chihuhua & 2 \\
\hline & & & Universidad de Sonora & 2 \\
\hline & & & Colegio de Michoacán & 1 \\
\hline & & & Universidad Autónoma de San Luis Potosí & 1 \\
\hline & & & Universidad de Colima & 1 \\
\hline & & & Universidad de Guadalajara & 1 \\
\hline & & & Universidad Pedagógica Nacional & 1 \\
\hline \multirow{5}{*}{$\begin{array}{l}\text { Gran } \\
\text { Bretaña }\end{array}$} & \multirow{5}{*}{9} & \multirow{5}{*}{8} & Glasgow Caledonian University & 4 \\
\hline & & & Universidad de Gales & 2 \\
\hline & & & The Open University & 1 \\
\hline & & & Universidad de Edimburgo & 1 \\
\hline & & & University of London & 1 \\
\hline \multirow{5}{*}{ Brasil } & \multirow{5}{*}{8} & \multirow{5}{*}{7} & Universidade de São Paulo & 3 \\
\hline & & & Universidade Católica de Brasília & 2 \\
\hline & & & Universidade Federal de Sergipe & 2 \\
\hline & & & $\begin{array}{l}\text { Escuela de Comunicaciones y Artes de la Universi- } \\
\text { dade de São Paulo }\end{array}$ & 1 \\
\hline & & & Universidade Federal do Rio Grande do Norte & 1 \\
\hline \multirow{2}{*}{ Alemania } & \multirow{2}{*}{5} & \multirow{2}{*}{5} & Catholic University Eichstaett-Ingolstadt & 3 \\
\hline & & & University of Wuerzburg & 2 \\
\hline
\end{tabular}




\begin{tabular}{|c|c|c|c|c|}
\hline \multirow{5}{*}{ Colombia } & \multirow{5}{*}{5} & \multirow{5}{*}{5} & Universidad EAFIT & 2 \\
\hline & & & Corporación Universitaria Lasallista & 1 \\
\hline & & & Universidad de La Sabana & 1 \\
\hline & & & Universidad de Los Andes & 1 \\
\hline & & & Universidad Santiago de Cali & 1 \\
\hline \multirow{3}{*}{ EEUU } & \multirow{3}{*}{4} & \multirow{3}{*}{4} & Southern Illinois University & 1 \\
\hline & & & Universidad de Maryland, College Park & 1 \\
\hline & & & University of California & 1 \\
\hline Perú & 4 & 4 & Universidad de Piura & 4 \\
\hline \multirow[t]{2}{*}{ Chile } & \multirow[t]{2}{*}{3} & \multirow[t]{2}{*}{3} & $\begin{array}{l}\text { Asociación Chilena de Investigadores en Comunica- } \\
\text { ción }\end{array}$ & 1 \\
\hline & & & Pontificia Universidad Católica de Chile & 1 \\
\hline Cuba & 2 & 2 & Universidad Central «Marta Abreu» de las Villas & 2 \\
\hline \multirow{2}{*}{ Venezuela } & \multirow{2}{*}{2} & \multirow{2}{*}{2} & Universidad Central de Venezuela & 1 \\
\hline & & & Universidad Universidad Bolivariana de Venezuela & 1 \\
\hline Italia & 2 & 2 & Universidad La Sapienza de Roma & 2 \\
\hline Japón & 1 & 1 & Aichi Prefectural University & 1 \\
\hline Países Bajos & 1 & 1 & University of Amsterdam & 1 \\
\hline
\end{tabular}

Entre las universidades españolas destacan la Universidad Rey Juan Carlos (URJC), la Universidad Complutense de Madrid (UCM) y la Universidad de Alicante (UA). De estas tres universidades nacionales, salvo en el caso de la UCM, en el resto (UA y URJC) priman las autoras sobre los autores.

Tabla 5: Distribución de autoras y autores por centro nacional

\begin{tabular}{|l|l|r|r|r|}
\hline $\begin{array}{c}\text { Comunidad } \\
\text { autónoma }\end{array}$ & \multicolumn{1}{|c|}{ Institución } & No & Mujeres & Hombres \\
\hline \multirow{4}{*}{$\begin{array}{l}\text { Comunidad } \\
\text { de Madrid }\end{array}$} & Universidad Rey Juan Carlos & 29 & 17 & 12 \\
\cline { 2 - 5 } & Universidad Complutense de Madrid & 28 & 13 & 15 \\
\cline { 2 - 5 } & CEU & 13 & 7 & 6 \\
\cline { 2 - 5 } & Universidad de Nebrija & 5 & 4 & 1 \\
\cline { 2 - 5 } & Centro Universitario Villanueva & 4 & 2 & 2 \\
\cline { 2 - 5 } & ESIC & 3 & 1 & 2 \\
\cline { 2 - 5 } & Universidad Carlos III & 3 & 3 & 0 \\
\cline { 2 - 5 } & Universidad Francisco de Vitoria & 2 & 1 & 1 \\
\cline { 2 - 5 } & Centro Universitario Cardenal Cisneros (UAH) & 1 & 1 & 0 \\
\cline { 2 - 5 } & Universidad Camilo José Cela & 1 & 1 & 0 \\
\cline { 2 - 5 } & Universidad Pontificia Comillas & 21 & 13 & 1 \\
\hline \multirow{1}{*}{$\begin{array}{l}\text { Comunidad } \\
\text { Valenciana }\end{array}$} & Universidad de Alicante & 14 & 9 & 5 \\
\cline { 2 - 5 } & Universidad Miguel Hernández & 9 & 5 & 4 \\
\cline { 2 - 5 } & Universitat Jaume I & & & 5 \\
\hline
\end{tabular}




\begin{tabular}{|c|c|c|c|c|}
\hline & Universitat de València & 5 & 1 & 4 \\
\hline & Universidad Politécnica de Valencia & 1 & 0 & 1 \\
\hline \multirow[t]{9}{*}{ Andalucía } & Universidad de Málaga & 17 & 7 & 10 \\
\hline & Universidad de Granada & 15 & 9 & 6 \\
\hline & Universidad de Huelva & 11 & 5 & 6 \\
\hline & Universidad de Sevilla & 11 & 8 & 3 \\
\hline & CEADE & 1 & 1 & 0 \\
\hline & Escuela Superior de Arte y Diseño de Andalucía & 1 & 1 & 0 \\
\hline & Universidad de Almería & 1 & 1 & 0 \\
\hline & Universidad de Cádiz & 1 & 0 & 1 \\
\hline & Universidad de Córdoba & 1 & 1 & 0 \\
\hline $\begin{array}{l}\text { Ámbito } \\
\text { nacional }\end{array}$ & UNED & 13 & 8 & 5 \\
\hline \multirow{3}{*}{$\begin{array}{l}\text { Castilla } \\
\text { y León }\end{array}$} & Universidad de Valladolid & 12 & 10 & 2 \\
\hline & Universidad de Salamanca & 2 & 0 & 2 \\
\hline & Universidad de Burgos & 1 & 1 & 0 \\
\hline Asturias & Universidad de Oviedo & 10 & 9 & 1 \\
\hline \multirow[t]{2}{*}{ Canarias } & Universidad de La Laguna & 8 & 2 & 6 \\
\hline & Universidad Europea de Canarias & 1 & 0 & 1 \\
\hline Navarra & Universidad de Navarra & 8 & 4 & 4 \\
\hline \multirow[t]{3}{*}{ Murcia } & Universidad de Murcia & 7 & 3 & 4 \\
\hline & Centro Autorizado ANMAVI de Murcia & 2 & 1 & 1 \\
\hline & Universidad Católica de Murcia & 2 & 1 & 1 \\
\hline Cantabria & Universidad de Cantabria & 6 & 6 & 0 \\
\hline \multirow[t]{5}{*}{ Cataluña } & Universidad Internacional de Cataluña & 6 & 3 & 3 \\
\hline & Universitat Ramón Llull & 6 & 3 & 3 \\
\hline & Universitat Autònoma de Barcelona & 5 & 4 & 1 \\
\hline & Universitat Pompeu Fabra & 5 & 1 & 4 \\
\hline & Universidad Autónoma de Barcelona & 2 & 2 & 0 \\
\hline La Rioja & Universidad Internacional de La Rioja & 6 & 2 & 4 \\
\hline Extremadura & Universidad de Extremadura & 4 & 2 & 2 \\
\hline \multirow[t]{3}{*}{ Galicia } & Universidade da Coruña & 4 & 4 & 0 \\
\hline & Universidad de Vigo & 4 & 3 & 1 \\
\hline & Universidad de Santiago de Compostela & 1 & 1 & 0 \\
\hline \multirow{2}{*}{$\begin{array}{l}\text { Castilla } \\
\text { La Mancha }\end{array}$} & Universidad de Castilla La Mancha & 2 & 2 & 0 \\
\hline & Junta Castilla La Mancha & 1 & 1 & 0 \\
\hline \multirow[t]{3}{*}{ País Vasco } & Universidad de Deusto & 2 & 2 & 0 \\
\hline & Universidad del País Vasco & 2 & 1 & 1 \\
\hline & Udako Euskal Unibertsitatea & 1 & 1 & 0 \\
\hline \multicolumn{2}{|l|}{ Total } & 340 & 190 & 150 \\
\hline
\end{tabular}

Fuente: elaboración propia 
En relación al idioma de los textos, prima el español $(85,7 \%)$ como único idioma, seguido del bilingüismo español-inglés $(6,9 \%)$, inglés $(4,9 \%)$, portugués $(2 \%)$ y de nuevo el bilingüismo, en este caso español-italiano $(0,4 \%)$. Así las cosas, solo el 9,7\% aporta las traducciones del texto original.

En el título, las palabras con mayor presencia son: comunicación, caso, social o sociales, análisis, estudio y digital o su derivado en plural, digitales.

\section{Gráfico 1: Frecuencia de aparición de palabras en título}

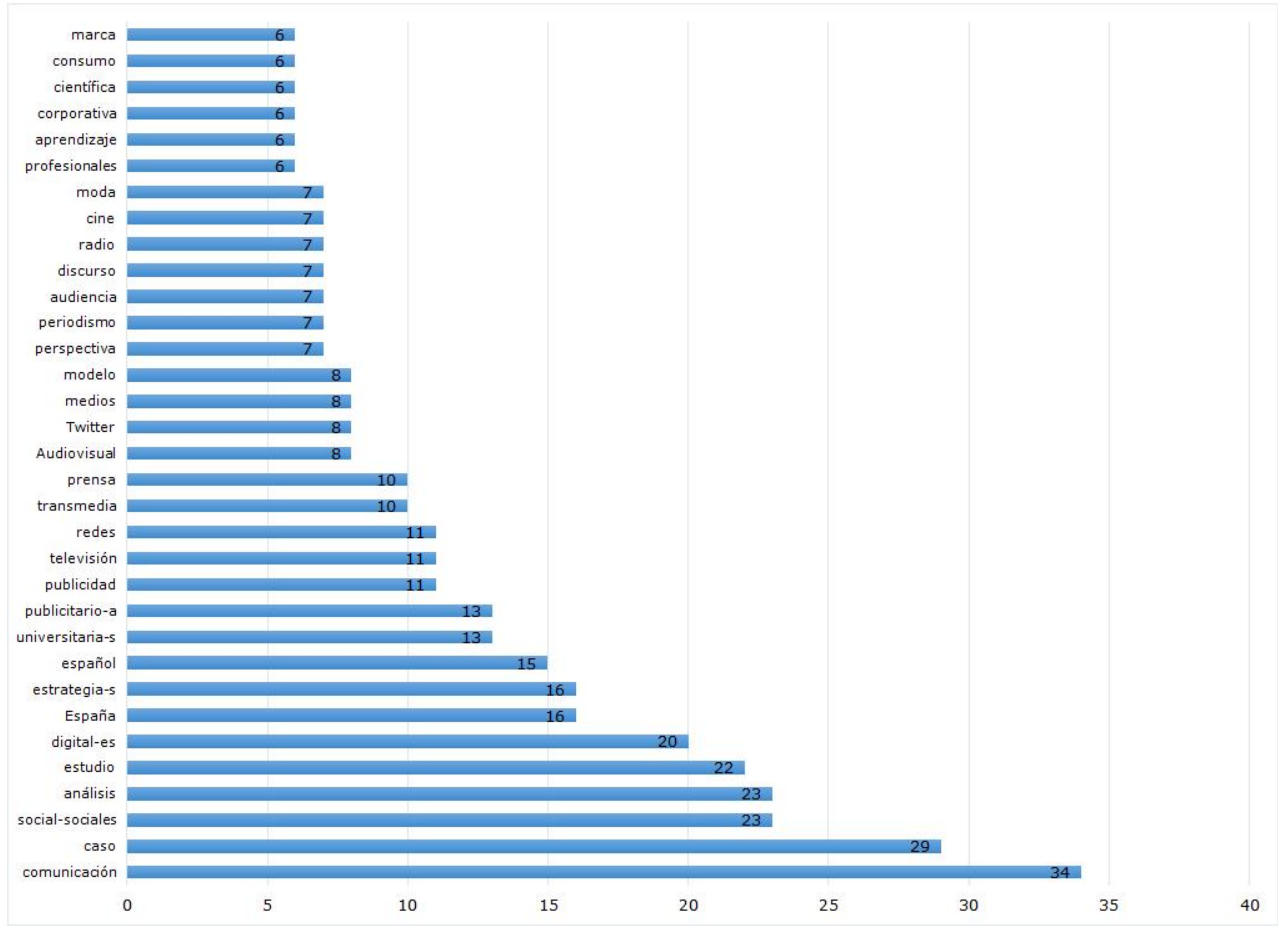

Fuente: elaboración propia a partir de nubedepalabras.es

En cambio, en las palabras clave en español, los términos más habituales son: social o sociales, comunicación, publicidad, redes, televisión, digital y periodismo. 


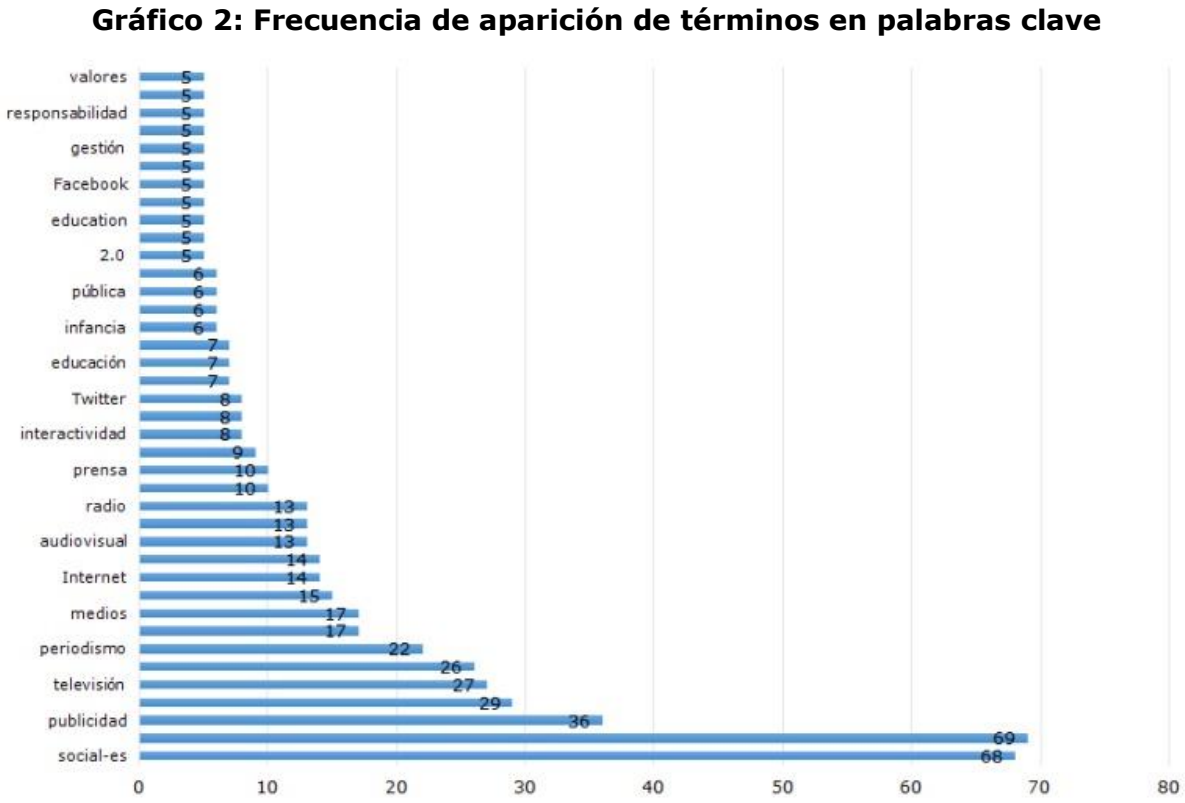

Fuente: elaboración propia a partir de nubedepalabras.es

En segundo lugar, con respecto al OE2, sí están presentes en Orcid el $79,7 \%$ de la autoría (56,8\% mujeres y $43,2 \%$ hombres) mientras que $20,3 \%$ no tiene perfil en Orcid (67,4\% mujeres y $32,6 \%$ hombres). Con respecto a la cumplimentación de sus perfiles - se ha de tener en cuenta que cabe la posibilidad de que la autoría se registre pero no cumplimente ni actualice su perfil- con respecto a la autoría que cuenta con perfil en Orcid, el 83,4\% sí lo tiene cumplimentado (54,6\% mujeres y $45,4 \%$ hombres) y el restante $16,6 \%$ no lo ha cumplimentado hasta la fecha del análisis (68,9\% mujeres y $31,1 \%$ hombres).

Atendiendo al análisis en Google Scholar se detectan los siguientes datos generales:

Tabla 6: Distribución de datos de Google Scholar

\begin{tabular}{|l|r|r|r|r|r|r|}
\hline & \multicolumn{3}{|c|}{ Total } & \multicolumn{3}{c|}{ Desde 2014 } \\
\cline { 2 - 7 } & $\mathbf{N}^{\circ}$ citas & Índice H & Índice H-10 & No todas citas $^{\text {Índice H }}$ & Índice H-10 \\
\hline Promedio & 594,5 & 39,3 & 11 & 324 & 7,4 & 7,7 \\
\hline Total & 163.485 & 10.818 & 3.012 & 89.088 & 2.029 & 2.118 \\
\hline
\end{tabular}

Fuente: elaboración propia 
De todas las variables relativas a Google Scholar, la más relevante es el número de citas. Al contrastar los datos relativos al número total de citas (163.485) con la variable género, se detecta que 95.413 citas las aportan los hombres $(58,4 \%)$ y las restantes 68.072 citas las mujeres $(41,6 \%)$.

Por otra parte, si se centra la atención sobre las autorías que mayor número de citas han recibido en su carrera investigadora, se obtienen los siguientes datos:

Tabla 7: Autores/as de RMC con más de 1000 en Google Scholar

\begin{tabular}{|l|r|}
\hline \multicolumn{1}{|c|}{ Autor/a } & No de citas \\
\hline Mark Deuze & 31.257 \\
\hline Juan-Bautista Martínez-Rodríguez & 11.826 \\
\hline Ignacio Aguaded & 10.401 \\
\hline Pablo Ramirez-Romero & 7.997 \\
\hline Ramón Salaverría & 5.042 \\
\hline Margarida-Maria Krohling-Kunsch & 4.984 \\
\hline Francisco García-García & 4.677 \\
\hline Alfonso Gutiérrez-Martín & 3.777 \\
\hline Roberto Aparici & 3.230 \\
\hline José-Alberto García-Avilés & 2.455 \\
\hline Cristina Ponte & 2.453 \\
\hline María-Luisa Humanes-Humanes & 2.230 \\
\hline María-Esther Del-Moral-Pérez & 2.143 \\
\hline João Canavilhas & 2.138 \\
\hline Andreu Casero-Ripollés & 2.109 \\
\hline José-Antonio Gómez-Hernández & 2.056 \\
\hline Rafael Alberto-Pérez & 1.841 \\
\hline Carmen Elboj-Saso & 1.755 \\
\hline Niall Sclater & 1.536 \\
\hline Carmen Marta-Lazo & 1.386 \\
\hline Lourdes Villalustre-Martínez & 1.268 \\
\hline Natalia González-Fernández & 1.216 \\
\hline Sara Osuna-Acedo & 1.215 \\
\hline José-Antonio Ortega-Carrillo & 1.207 \\
\hline Araceli Castelló-Martínez & 1.083 \\
\hline Carmen Costa-Sánchez & 1.043 \\
\hline Miguel Ángel Ortiz-Sobrino & \\
\hline
\end{tabular}

Fuente: elaboración propia

Dentro de las autorías con mayor repercusión en Google Scholar, el $51,9 \%$ son hombres y el $48,1 \%$ son mujeres. Se percibe que, aunque las muje- 
res son las principales autoras en RMC, la comunidad académica tiende a citar más a los hombres, aunque la diferencia $(3,8 \%)$ es escasa e irrelevante.

En este sentido se contrastan las autorías con respecto a los cinco artículos publicados en $R M C$ con mayor número de citas (tabla 8). Destaca el texto de Aparici y Osuna-Acedo (99 citas), seguido del de Ramón Salaverría (47), el de Del-Pino-Romero y Castelló-Martínez (46), el de Mark Deuze (34) y el de Leticia Rodríguez-Fernández (32). Estos datos evidencian que de los cinco artículos más citados de $R M C$, tres están firmados por mujeres en coautoría hombre-mujer, en coautoría femenina y en solitario.

Tabla 8: Artículos RMC con mayor número de citas en Google Scholar

\begin{tabular}{|l|l|c|}
\hline \multicolumn{1}{|c|}{ Título: año, volumen (número) } & \multicolumn{1}{|c|}{ Autor/a } & $\begin{array}{c}\text { No de } \\
\text { citas }\end{array}$ \\
\hline La cultura de la participación: 2013, 4(2) & Roberto Aparici & 99 \\
\cline { 2 - 2 } & Sara Osuna-Acedo & 47 \\
\hline $\begin{array}{l}\text { Tipología de los cibermedios periodísticos: } \\
\text { bases teóricas para su clasificación: 2017, 8(1) }\end{array}$ & Ramón Salaverría & 46 \\
\hline $\begin{array}{l}\text { La comunicación publicitaria se pone de moda: } \\
\text { branded content y fashion films: 2015, 6(1) }\end{array}$ & Cristina Del Pino-Romero & Araceli Castelló-Martínez \\
\hline $\begin{array}{l}\text { Considerando el futuro del periodismo digital: } \\
\text { 2017, 8(1) }\end{array}$ & Mark Deuze & 34 \\
\hline $\begin{array}{l}\text { Smartphones y aprendizaje: el uso de Kahoot } \\
\text { en el aula universitaria: 2017, 8(1) }\end{array}$ & Leticia Rodríguez-Fernández & 32 \\
\hline
\end{tabular}

Fuente: elaboración propia

En relación al OE3, en primer lugar, de los ocho componentes del equipo editorial de $R M C, 7(87,5 \%)$ son mujeres y uno es hombre $(12,5 \%)$. En segundo lugar, el comité internacional de edición está formado once mujeres $(64,7 \%)$ y seis hombres $(35,3 \%)$. En tercer lugar, el comité científico está compuesto por 34 mujeres $(59,6 \%)$ y 23 hombres $(40,4 \%)$. Y, por último, en relación a los/as 382 revisores/as que al menos han revisado un texto para $R M C$, priman las mujeres (235 y 61,5\%) sobre los hombres (147 y 38,5\%).

\section{Conclusiones}

El estudio realizado ha permitido indagar el perfil de autoría y de colaboración en $R M C$ y su relación con el género, así como la repercusión de la autoría y artículos de la revista presentes en Google Scholar.

En relación a la autoría (OE1), la revista revela una presencia ostensible de mujeres, tanto en la coordinación de monográficos y autoría de artículos científicos como también en su colaboración en los distintos consejos de edición y revisión (OE3), siendo los resultados relativos al equipo de revisores/as dispares a los señalados por Schottdorf, Neef \& Battaglia (2017). A 
diferencia de Blackburn \& Lawrence (1995), Creamer (1998), Dinauer \& Ondeck (1999), Buffalino-Roland \& Fontanesi-Seime (1996) y Schneider (1998), la mayor parte de la autoría de $R M C$ son mujeres, doctoras y firman con alto índice de coautoría, aunque como autoría única sigue prevaleciendo la figura del hombre y como primer firmante la mujer, en contraposición a lo recogido por Wilson (2012), compartiendo en cierta medida el protagonismo del artículo pero tendiendo la figura del hombre hacia el individualismo en su rol de productor científico.

La procedencia nacional (y las universidades URJC y UCM) prima sobre la internacional (y las universidades UBA.ar, UNDAV.ar, UNLZ.ar y UNR.ar). Se evidencia una clara procedencia latinoamericana (Argentina, Brasil, Chile, Colombia, Cuba, Ecuador, México, Perú y Venezuela). El español prima como idioma principal seguido de los artículos bilingües español-inglés, aunque esto puede cambiar a futuro ya que desde enero de 2019 la traducción se hace obligatoria en $R M C$. La cada vez más frecuente coordinación internacional de los monográficos y el incremento de colaboraciones foráneas también lo auguran.

Se detecta una gran dispersión institucional de la autoría, especialmente en el ámbito internacional, no tanto dentro de España, donde las 10 primeras universidades con mayor representación en la autoría de $R M C$ copan el 51,8\% de las autorías.

Aunque no se ha podido relacionar la variable género con respecto a los términos más frecuentes en títulos y resúmenes, dado que estas son variables que se analizan en función del artículo y no de la autoría, el concepto comunicación prima tanto en los títulos como en las palabras clave de los artículos, dada el área temática de la revista y el hecho de no estar especializada en ninguna de las ramas (periodismo, comunicación audiovisual ni comercial), si bien esta última (comunicación comercial o persuasiva) sobresale.

Además, resulta destacable la importancia del estudio de caso, teniendo en cuenta el uso de la propia palabra 'caso' en los títulos, cuya frecuencia la sitúa en segunda posición. Esto invita al equipo investigador a afirmar que el concepto de comunicación, unido al estudio de caso, suponen una tendencia evidente en la revista en cuestión, que podría ser extrapolable a otras revistas del área, previo estudio. Otros aspectos destacables que permiten evidenciar las tendencias más representativas en el contenido de la revista son las frecuencias relativas a los conceptos 'digital' y 'estrategia' que ocupan puestos referentes en los títulos, indicando así, la tendencia detectada en el contenido de las publicaciones de la revista.

En relación al OE2, se evidencia una alta implicación participativa de la autoría en Orcid y Google Scholar. Con respecto a esta última, a diferencia de la 
perspectiva productora, prima la figura del hombre con respecto al rol de liderazgo, ya que los hombres reciben más citas en general que las mujeres. Pero si cambiamos la unidad de análisis de la autoría a los artículos de $R M C$ con mayor repercusión en Google Scholar, los datos evidencian un creciente protagonismo de la mujer sobre el hombre y, además, en las distintas alternativas de participación en el artículo: coautoría hombre-mujer, coautoría mujer-mujer y autoría única.

Nuevos retos se imponen necesarios. En primer lugar, en $R M C$ : incorporar mayor porcentaje de autoría internacional. Y, en segundo lugar, para el equipo investigador: ampliar este estudio de género incorporando más revistas del área de Comunicación, de Ciencias Sociales o de forma transversal; analizar la obtención de financiación pública y privada para la investigación y difusión del conocimiento (García de Cortázar y García de León, 2001) y prestar más atención al comité de evaluación (de revisiones) en línea a lo realizado por Schottdorf, Neef \& Battaglia (2017). En suma, se trata de realizar un homenaje y reconocimiento público a la mujer (Vega y Santos, 2010) en el ámbito académico, científico y editorial; una consideración que la literatura consultada ha evidenciado como necesaria tanto desde la perspectiva histórica como social.

\section{Referencias bibliográficas}

AGUDO, Y. (2006). El lado oscuro de la mujer en la investigación científica: ¿es la ciencia una 'empresa' masculina? Cuestiones de género: de la igualdad y la diferencia, 2006, 1, 15-51. http://dx.doi.org/10.18002/cg.v0i1.3852

AGUILAR, C.; AlONSO, M.J.; MELGAR, P. y MolinA, S. (2009). Violencia de género en el ámbito universitario: medidas para su superación. Pedagogía social: Revista Interuniversitaria, 16, 85-94. Recuperado el 26 de septiembre de 2019 de https: / / bit.ly / 2IPoMo3

ALCALÁ, P. (2006). Españolas en el CSIC. Presencia y status de las mujeres en la investigación científica española 1940-1993. En Ortiz, T. y Becerra, C. (coord.). Mujeres de ciencias. Mujer, feminismo y ciencias naturales, experimentales y tecnológicas (pp.61-76). Granada: Instituto de la Mujer de la Universidad de Granada.

ALIC, M. (1981). El legado de Hipatia. Historia de las mujeres en la ciencia desde la Antigüedad hasta fines del siglo XIX. México: Siglo XXI.

ALMODÓVAR, M.A. (1996). Mujer y ciencia en Iberoamérica. Invisibilidad y familia. En Ortiz, T. y Becerra, C. (coord.). Mujeres de ciencias. Mujer, feminismo y ciencias naturales, experimentales y tecnológicas (pp. 83-96). Granada: Instituto de la Mujer de la Universidad de Granada. 
BlACKBURN, R.T. \& LAWRENCE, J.H. (1995). Faculty at Work: Motivation, Expectation, Satisfaction. Baltimore: John Hopkins University Press.

BorsuK, R.M.; AARsSEN, L.W.; Budden, A.E.; KoricheVA, J.; LEIMU, R.; TREGEnZA, T. \& LORTIE, C.J. (2009). To name or not to name: the effect of changing author gender on peer review. Bio Science, 59, 985-989. https://doi.org/10.1525/bio.2009.59.11.10

BuCKLEY, H.L.; SCILIGO, A.R.; ADAIR, K.L.; CASE, B.S. \& MONKS, J.M. (2014). Is there gender bias in reviewer selection and publication success rates for the 'New Zealand Journal of Ecology'? New Zealand Journal of Ecology. 38, 335339. Recuperado el 26 de septiembre de 2019 de https:// bit.ly/33PjsWb Budden, A.; TregenzA, T.; AARssen, L.; Koricheva, J.; Leimu, R. \& LorTiE, C. (2008). Double-blind review favours increased representation of female authors. Trends in Ecology \& Evolution, 23, 4-6.

https://doi.org/10.1016/j.tree. 2007.07.008

Buffalino-Roland, C. \& Fontanesi-SEime, M. (1996). Women Counselor Educators: A Survey of Publication Activity. Journal of Counseling \& Development, 74, 490-499.

https://doi.org/10.1002/j.1556-6676.1996.tb01898.x

Clair, R. (ed.) (1996). La formación científica de las mujeres. ¿Por qué hay tan pocas científicas? Madrid: Los Libros de la Catarata.

Comunicar (2019). Ranking de revistas ESCI de comunicación [en línea].

Recuperado el 27 septiembre de 2019 de https:/ / bit.ly/2X2r975

Creamer, E.G. (1998). Assessing Faculty Publication Productivity. Issues of

Equity. ASHE-ERIC Higher Education Report. Recuperado el 26 de septiembre de 2019 de https://bit.ly/2HgT91N

Demarest, B.; FreEmAn, G. \& Sugimoto, C.R. (2014). The reviewer in the mirror: examining gendered and ethnicized notions of reciprocity in peer review. Scientometrics, 717-735. https://doi.org/10.1007/s11192-014-1354-z

Dialnet Métricas (2018). Indicadores de la producción científica (Comunicación) [en línea]. Recuperado el 27 de septiembre de 2019 de https: / / bit.ly/2TOY7as

DiNAUER, L.D. \& ONDECK, K.E. (1999). Gender and Institutional Affiliationas Determinants of Publishing in Human Communications Research. Human Communication Research, 25, 548-568.

https://doi.org/10.1111/j.1468-2958.1999.tb00461.x

FONG, K.; HUNG, M.C. \& LI, J.K.K. (2005). Asian scientists and the 'glass ceiling'. Science, 310, 1767-1768.

https://doi.org/10.1126/science.310.5755.1767b

FoX, C.W.; BuRnS, C.S. \& MEYER, J.A. (2016). Editor and reviewer gender influence the peer review process but not peer review outcomes at an 
ecology journal. Functional Ecology, 30, 140-153.

https://doi.org/10.1111/1365-2435.12529

GARcía De CortázAR, M. y GARCÍA DE LEÓN, M.A. (2001). Las Académicas (profesorado universitario y género). Madrid: Instituto de la Mujer.

GILBERT, J.R.; WILLIAMS, E.S. \& LUNDBERG, G.D. (1994). Is there gender bias in JAMA's peer review process? JAMA, 272, 139-142. https://doi.org/10.1001/jama.1994.03520020065018

GonzÁLEZ, M. y PÉREZ, E. (2002). Ciencia, Tecnología y Género. Revista Iberoamericana de Ciencia, tecnología y sociedad de la innovación, 2, s.p. Recuperado el 26 de septiembre de 2019 de https: / / bit.ly/30n6FrV Google Académico. (2009). Citas de Revista Mediterránea de Comunicación /Mediterranean Journal of Communication [en línea]. Recuperado el 25 de enero de 2020 de https: / / bit.ly/2zes9LH

GuIL BozAL, A. (2007). Docentes e investigadoras en las universidades españolas: visibilizando techos de cristal. Revista de investigación educativa. 25, 111-131. Recuperado el 26 septiembre de 2019 de https: / / bit.ly / 2P5Boc0

Handley, G.; FrantZ, C.M.; Kocovsky, P.M.; De VRIES, D.R.; CoOKE, S.J, \& Claussen, J. (2015). An examination of gender differences in the American Fisheries Society peer-review process. Fisheries, 40. 442-451.

https: / / doi.org/10.1080/03632415.2015.1059824

Helmer, M.; SCHOTTDORF, M.; NEEF, A. \& BATTAGLiA, D. (2017). Gender bias in scholarly peer review. Elife, 6, s.p.

https: / / doi.org/10.7554/eLife.21718.001

Instituto De La Mujer. (2009). Las mujeres en el Sistema educativo. Madrid: Instituto de la Mujer (Ministerio de Igualdad). Recuperado el 24 de septiembre de 2019 de https: / / bit.ly/2kSrR9z

Instituto Nacional De Estadística. (2019). Mujeres en el profesorado por enseñanza que imparten [en línea]. Recuperado el 24 de septiembre de 2019 de https: / / bit.ly/2mjj6G6

Knobloch-Westerwick, S.; GLYnN, C.J. \& Huge, M. (2013). The Matilda effect in science communication an experiment on gender bias in publication quality perceptions and collaboration interest. Science Communication, 35, 603-625. https: //doi.org/10.1177/1075547012472684

LARIVIÈRE, V.; NI, C.; GingraS, Y.; CRONIN, B. \& SugIMOTO, C.R. (2013). Bibliometrics: Global gender disparities in science. Nature, 7459, 211-213. https: / / doi.org/10.1038/504211a

LLOYD, M.E. (1990). Gender factors in reviewer recommendations for manuscript publication. Journal of Applied Behavior Analysis. 23, 539-543. https: / / doi.org/10.1901/jaba.1990.23-539 
MAGAllón PoRTOLÉS, C. (1999). Pioneras españolas en las ciencias: las mujeres del Instituto Nacional de Física y Química. Madrid: CSIC.

MATHEWS, A.L. \& ANDERSEN, K. (2001). A gender gap in publishing? Women's representation in edited political science books. PS: Political Science \& Politics, 34, 143-147. https://doi.org/10.1017/S1049096501000221

PEÑASCO, R. (2005). Mobbing en la universidad: ¿tesis, birretes, togas... y cum laude en acoso? Madrid: Adhara.

Redib (2019). Ranking de Revistas REDIB [en línea]. Recuperado el 26 de septiembre de 2019 de https: / / bit.ly/2L3Bmv7

SANTESMASES, M.J. (2000). Mujeres científicas en España (1940-1970): profesionalización y modernización social. Madrid: Instituto de la Mujer.

SchneIDER, A. (1998). Why Don't Women Publish as Much as Men? Some Blame Inequity in Academe; Others Say Quantity Doesn't Matter. The Chronicle of Higher Education, 45, 14-16. Recuperado el 26 de septiembre de 2019 de https: / / bit.ly/2Muhbum

SQUIRES, J. (2008). Diversity mainstreaming: dépasser les approaches technocratiques et d'addiction des inégalités. Cahiers du Genre, 44, 73-94. https: / / doi.org/10.3917/cdge.044.0073

VALLS, R. (2008). Violencia de género en las Universidades españolas. Memoria final e informe de investigación. Barcelona: Ministerio de Igualdad, Instituto de la Mujer.

Vega NAVARro, A. y SANTOS VEGA, J.D. (2010). La mujer en la Universidad de La Laguna. Qurriculum: Revista de Teoría, Investigación y Práctica Educativa, 23, 131-154. Recuperado el 26 de septiembre de 2019 de https: / / bit.ly/2L07xwe

WILSON, R. (2018). Scholarly Publishing's Gender Gap. Chronicle of Higher Education [en línea]. Recuperado el 27 de septiembre de 2019 de https: / / bit.ly/31RKKtf

WoODWARD, A.E. (2008). Est-il trop tard pour une approche intégrée de l'égalité? Le point sur la situation à Bruxelles. Cahier du Genre, 44, 95-116. https://doi.org/10.3917/cdge.044.0095 